TAIWANESE JOURNAL OF MATHEMATICS

Vol. 6, No. 2, pp. 187-204, June 2002

\title{
EXISTENCE OF STRONG SOLUTIONS TO SOME QUASILINEAR ELLIPTIC PROBLEMS ON BOUNDED SMOOTH DOMAINS
}

\author{
Tsang-Hai Kuo and Yeong-Ju Chen
}

Abstract. We consider the following quasilinear elliptic problems in a bounded
smooth domain $Z$ of $\mathbb{R}^{N}, N \geq 3$ :
$\begin{cases}L u=\sum_{i, j=1}^{N} a_{i j}(x, u) \frac{\partial^{2} u}{\partial x_{i} \partial x_{j}}+\sum_{i=1}^{N} b_{i}(x, u) \frac{\partial u}{\partial x_{i}}+c(x, u) u=f(x) & \text { in } Z, \\ u=0 & \text { on } \partial Z,\end{cases}$

where $f(x) \in L^{p}(Z)$ and all the coefficients $a_{i j}, b_{i}, c$ are Carathédory functions. Suppose that $a_{i j} \in C^{0,1}(\bar{Z} \times \mathbb{R}), a_{i j}, \partial a_{i j} / \partial x_{i}, \partial a_{i j} / \partial r$, $b_{i}, c \in L^{\infty}(Z \times \mathbb{R}), c \leq 0$ for $i, j=1, \ldots N$ and the oscillations of $a_{i j}=a_{i j}(x, r)$ with respect to $r$ are sufficiently small. A global estimate for a solution $u \in W^{2, p}(Z) \cap W_{0}^{1, p}(Z)$ is established and the existence of a strong solution $u \in W^{2, p}(Z) \cap W_{0}^{1, p}(Z)$ is proved for $p>N$.

Furthermore, we replace $f(x)$ by $f(x, r, \xi)$ which is defined on $Z \times \mathbb{R} \times \mathbb{R}^{N}$ and is a Carathédory function. Assume that

$$
|f(x, r, \xi)| \leq C_{0}+h(|r|)|\xi|^{\theta}, \quad 0 \leq \theta<2,
$$

where $C_{0}$ is a nonnegative constant, $h(|r|)$ is a locally bounded function, and $-c \geq \alpha_{0}>0$ for some constant $\alpha_{0}$. We prove the existence of solution $u \in W^{2, p}(Z) \cap W_{0}^{1, p}(Z)$ for the equation $L u=f(x, u, \nabla u)$.

Received March 3, 2000.

Communicated by S.-B. Hsu.

2000 Mathematics Subject Classification: Primary 35D05, 35J25; Secondary 46E35.

Key words and phrases: Quasilinear elliptic problem, strong solution.

Partially supported by the National Science Council of R.O.C. under Project NSC88-2115-M009-012 and NSC89-2115-M009-013. 


\section{INTRODUCTION}

Let $\Omega$ be a bounded $C^{1,1}$ domain in $\mathbb{R}^{N}, N \geq 3$, and $L$ be the following elliptic operator in the general form:

$$
L u=\sum_{i, j=1}^{N} a_{i j}(x, u) \frac{\partial^{2} u}{\partial x_{i} \partial x_{j}}+\sum_{i=1}^{N} b_{i}(x, u) \frac{\partial u}{\partial x_{i}}+c(x, u) u, \quad x \in \Omega
$$

We study the existence of strong solutions to the following problems:

$$
\begin{cases}L u=f(x) & \text { in } \Omega, \\ u=0 & \text { on } \partial \Omega\end{cases}
$$

where $f \in L^{p}(\Omega)$, and

$$
\left\{\begin{array}{lr}
L u=f(x, u, \nabla u) & \text { in } \Omega, \\
u=0 & \text { on } \partial \Omega,
\end{array}\right.
$$

where $f(x, r, \xi)$ has less than quadratic growth in $\xi$. All the coefficient functions $a_{i j}, b_{i}, c$ and the function $f(x, r, \xi): \Omega \times \mathbb{R} \times \mathbb{R}^{N} \rightarrow \mathbb{R}$ are Carathédory functions, that is, the function $x \mapsto f(x, r, \xi)$ is measurable for all $(r, \xi) \in \mathbb{R} \times \mathbb{R}^{N}$ and the function $(r, \xi) \mapsto f(x, r, \xi)$ is continuous for a.e $x \in \Omega$.

The basic idea is to consider a mapping $F$ defined on $W^{2, p}(\Omega) \cap W_{0}^{1, p}(\Omega)$ by letting $u=F(v)$ be the unique solution in $W^{2, p}(\Omega) \cap W_{0}^{1, p}(\Omega)$ to the linear Dirichlet problem:

$$
\begin{cases}L_{v} u=\sum_{i, j=1}^{N} a_{i j}(x, v) \frac{\partial^{2} u}{\partial x_{i} \partial x_{j}}+\sum_{i=1}^{N} b_{i}(x, v) \frac{\partial u}{\partial x_{i}}+c(x, v) u=f(x) & \text { in } \Omega \\ u=0 & \text { on } \partial \Omega .\end{cases}
$$

The unique solvability of problem (1.2) is guaranteed by the linear existence result $[1$, p. 241] under appropriate coefficient conditions. We notice that $F$ is welldefined for $p>N / 2$. We shall then obtain solutions of problem (1.1) by finding fixed points of $F$.

The regularity theorem of Agmon-Douglis-Nirenberg [2] asserts that

$$
\|u\|_{W^{2, p}} \leq C\left(\|u\|_{L^{p}}+\left\|L_{v} u\right\|_{L^{p}}\right),
$$

where $C$ is a constant dependent on the moduli of continuity of the coefficients $a_{i j}(x, v(x))$ on $\bar{\Omega}$, etc. If $a_{i j}(x, r)=a_{i j}(x)$, then the constant $C$ in (1.3) is independent of $v$ and by [1, p. 243], there exists a constant $C$ independent of $v$ such that

$$
\|u\|_{W^{2, p}} \leq C\left\|L_{v} u\right\|_{L^{p}}=C\|f\|_{L^{p}} .
$$


According to the uniqueness of problem (1.2), $F$ is a continuous mapping in the topology of $W^{1, p}(\Omega)$ (Lemma 2.2.1). From (1.4), $\|u\|_{W^{2, p}} \leq K$ for some constant $K>0$. Let

$$
\mathcal{K}=\left\{v \in W^{2, p}(\Omega) \cap W_{0}^{1, p}(\Omega) \mid \quad\|v\|_{W^{2, p}(\Omega)} \leq K\right\} .
$$

By the Sobolev imbedding theorem, $\mathcal{K}$ is a compact convex set in $W^{1, p}(\Omega)$. Applying the Schauder fixed point theorem, we then obtain a solution to problem (1.1).

In the general case $a_{i j}=a_{i j}(x, r)$, the essence of our consideration is to establish estimate (1.3) for which the constant $C$ is independent of $v$. If $\Omega=B$ is a ball in $\mathbb{R}^{N}$, it has been shown in [3, Proposition 3.1.2] that

$$
\|u\|_{W^{2, p}(B)} \leq C\left(\|u\|_{L^{p}(B)}+\left\|L_{v} u\right\|_{L^{p}(B)}\right),
$$

where $C$ is independent of $v$. In Section 2, we intend to transform the coordinates in a bounded smooth domain $Z$ into a ball $\mathrm{B}$. By imposing stronger conditions on $a_{i j} \in C^{0,1}(\bar{Z} \times \mathbb{R})$ so that the oscillations with respect to $r$ are sufficiently small, we have the same estimate of (1.5) in Proposition 2.1.1. Together with the maximum principle of A. D. Aleksandrove [1, p. 220],

$$
\sup _{Z}|u| \leq C\|f\|_{L^{N}(Z)},
$$

where $C$ is a nonnegative constant, we show that $u$ is $W^{2, p}(Z)$ bounded. By the same argument as above, the existence of strong solutions to problem (1.1) is proved in Proposition 2.2.2.

Based on the preceding results, in Section 3, we further study the existence of strong solutions to the following quasilinear elliptic problem:

$$
\left\{\begin{array}{lr}
L u=f(x, u, \nabla u) & \text { in } Z, \\
u=0 & \text { on } \partial Z .
\end{array}\right.
$$

Suppose that

$$
-c \geq \alpha_{0}>0, \quad \text { for some constant } \alpha_{0},
$$

and $f(x, r, \xi)$ is a Carathédory function which satisfies

$$
|f(x, r, \xi)| \leq C_{0}+h(|r|)|\xi|^{\theta},
$$

where $C_{0}$ is a nonnegative constant, $h$ is a locally bounded function and $0 \leq \theta<2$. Then problem (1.6) has a strong solution $u \in W^{2, p}(Z) \cap W_{0}^{1, p}(Z)$ provided that the oscillations of $a_{i j}$ with respect to $r$ are sufficiently small. The result will be shown in Theorem 3.1. To prove the theorem, we consider the approximation of problem (1.6). Denote the corresponding solutions by $\left(u_{n}\right)$ (derived in Lemma 3.2). We first 
obtain a $L^{\infty}$ bound of subsequence of $\left(u_{n}\right)$ (Lemma 3.3), still relabeled as $\left(u_{n}\right)$, and then establish a $W^{2, p}$ bound of $\left(u_{n}\right)$ (Lemma 3.4). Finally, we pass the limit to verify that the limit $u$ of $\left(u_{n}\right)$ is a $W^{2, p}(Z) \cap W_{0}^{1, p}(Z)$ solution of problem (1.6).

The following notations are used in this paper. We denote by $\Omega, \partial \Omega, \mathrm{B}, Z$, and $\nabla u$ the open set in $\mathbb{R}^{N}$, the boundary of $\Omega$, the ball in $\mathbb{R}^{N}$, the bounded smooth domain in $\mathbb{R}^{N}$, and the gradient of $u$, respectively. We define $C^{k, \alpha}(\bar{\Omega})$ to be the space of functions in $C^{k}(\bar{\Omega})$ consisting of function whose $k$ th order partial derivatives are uniformly Hölder continuous with exponent $\alpha$ in $\Omega, 0<\alpha \leq 1$, and $C_{0}^{\infty}$ to be the space of functions in $C^{\infty}(\Omega)$ with compact support in $\Omega$. Let $W^{m, p}(\Omega):=\left\{u \in L^{P}(\Omega) \mid\right.$ weak derivatives $D^{\alpha} u \in L^{P}(\Omega)$ for all $\left.|\alpha| \leq m\right\}$ and $W_{0}^{m, p}$ be the closure of $C_{0}^{\infty}(\Omega)$ in $W^{m, p}(\Omega)$. We denote by $D^{2} u=\left[D_{i j} u\right]$ the Hessian matrix of second derivatives $D_{i j} u\left(=\partial^{2} u / \partial x_{i} \partial x_{j}\right), i, j=1,2, \ldots, N$.

\section{The Existence of Strong Solutions in Bounded Smooth Domains}

Let $Z$ be a bounded domain in $\mathbb{R}^{N}$ which is $C^{1,1}$ diffeomorphic to a ball B in $\mathbb{R}^{N}, \psi$ be a $C^{1,1}$ diffeomorphism from $\bar{Z}$ onto a ball $\bar{B}$ in $\mathbb{R}^{N}$ and $L$ be a second-order elliptic operator of the following form:

$$
L u=\sum_{i, j=1}^{N} a_{i j}(x, u) \frac{\partial^{2} u}{\partial x_{i} \partial x_{j}}+\sum_{i=1}^{N} b_{i}(x, u) \frac{\partial u}{\partial x_{i}}+c(x, u) u \quad x \in Z .
$$

In this section, we consider the Dirichlet problem for $L u=f(x)$ with $f \in L^{p}(Z)$. A global $W^{2, p}$ estimate for $\mathrm{u} \in W^{2, p}(Z) \cap W_{0}^{1, p}(Z)$ is also established and is used to prove the existence of a strong solution $\mathrm{u} \in W^{2, p}(Z) \cap W_{0}^{1, p}(Z)$.

\subsection{Global Estimate}

An operator $L$ in (2.0) is said to be uniformly elliptic in $\Omega$ if there exists a constant $\lambda>0$ such that

$$
\sum_{i, j=1}^{N} a_{i j}(x, r) \xi_{i} \xi_{j} \geq \lambda|\xi|^{2} \quad \text { for }(r, \xi) \in \mathbb{R} \times \mathbb{R}^{N} \text { and a.e. } x \in \Omega .
$$

For a fixed point $x \in \mathbb{R}^{N}$, we denote by osc $a_{i j}(x, r)$ the oscillation of $a_{i j}$ with respect to $\mathrm{r}$ in $\mathbb{R}$, that is, osc $a_{i j}(x, r)=\sup \left\{\left|a_{i j}\left(x, r_{1}\right)-a_{i j}\left(x, r_{2}\right)\right| \mid r_{1}, r_{2} \in \mathbb{R}\right\}$, and let

$$
\operatorname{osc} a(x, r)=\max _{1 \leq i, j \leq N} \text { osc } a_{i j}(x, r) \text {. }
$$

For $v \in W^{2, p}(\Omega) \cap W_{0}^{1, p}(\Omega)$, let

$$
L_{v} u=\sum_{i, j=1}^{N} a_{i j}(x, v) \frac{\partial^{2} u}{\partial x_{i} \partial x_{j}}+\sum_{i=1}^{N} b_{i}(x, v) \frac{\partial u}{\partial x_{i}}+c(x, v) u .
$$


Recall the Marcinkiewicz Interpolation and Calderon-Zygmund theorems. The $L^{p}$ estimate for a solution $u \in W_{0}^{2, p}(\Omega)$ of Poisson's equation in a domain $\Omega[1, \mathrm{p}$. $235]$ is given by

$$
\left\|D^{2} u\right\|_{L^{p}(\Omega)} \leq K\|\Delta u\|_{L^{p}(\Omega)},
$$

where $K=K(N, P)$ is a nonnegative constant. Notice that if $\Omega$ is a unit ball $B$, the global estimate of the $W^{2, p}(B)$ norm on $u$ is given by [3, Proposition 3.1.2]

$$
\|u\|_{W^{2, p}(B)} \leq C\left(\|u\|_{L^{p}(B)}+\left\|L_{v} u\right\|_{L^{P}(B)}\right),
$$

where $C$ is a constant (independent of $v$ ) dependent on $N, P, \lambda, \Lambda, \partial B, B$ and the moduli of continuity of the coefficients $a_{i j}(x, r)$ with respect to $\mathrm{x}$ on $\bar{B}$, $\left|a_{i j}\right|,\left|b_{i}\right|,|c| \leq \Lambda$ and osc $a(x, r)<\lambda / 4 K \forall x \in B$, osc $a(x, r)<\lambda / 8 N^{2} K \forall x \in$ $\partial B, K$ is a constant by (2.1.2). We start to establish a similar $W^{2, p}(Z)$ estimate as (2.1.3) for a bounded smooth domain $Z$ of $\mathbb{R}^{N}$. A global $W^{2, p}(Z)$ estimate can be derived by using the diffeomorphism to transform the coordinates to $B$ and then applying the $W^{2, p}(B)$ estimate. Therefore, we have the following proposition.

Proposition 2.1.1. Let $Z$ be a bounded smooth domain in $\mathbb{R}^{N}$ and the coefficients of L satisfies

$$
a_{i j} \in C^{0,1}(\bar{Z} \times \mathbb{R}), b_{i}, c \in L^{\infty}(Z \times \mathbb{R}),\left|a_{i j}\right|,\left|b_{i}\right|,|c| \leq \Lambda,
$$

where $\Lambda$ is a positive constant, $i, j=1, \ldots, N$. Assume that there exists a $C^{1,1}$ diffeomorphism $\psi$ from $\bar{Z}$ onto unit ball $\bar{B}$ in $\mathbb{R}^{N}, \psi(\partial Z)=\partial B$,

$$
G=\left[\begin{array}{ccc}
\frac{\partial \psi_{1}}{\partial x_{1}} & \cdots & \frac{\partial \psi_{1}}{\partial x_{N}} \\
\vdots & \ddots & \vdots \\
\frac{\partial \psi_{N}}{\partial x_{1}} & \cdots & \frac{\partial \psi_{N}}{\partial x_{N}}
\end{array}\right]
$$

$$
\begin{gathered}
\operatorname{osc} a(x, r) \leq \frac{\lambda}{4\left(\frac{\beta}{\alpha}\right) K} \quad \forall x \in Z, \\
\operatorname{osc} a(x, r) \leq \frac{\lambda}{8 N^{2}\left(\frac{\beta}{\alpha}\right) K} \quad \forall x \in \partial Z,
\end{gathered}
$$

where

$$
\xi\left(G G^{T}\right) \xi^{T} \geq \alpha|\xi|^{2} \quad \text { for some constant } \alpha>0 \text { ([4, P.539]), }
$$

$$
\beta=\max _{x \in \bar{Z}, 1 \leq i, j \leq N} \sum_{r, s}^{N}\left|\frac{\partial \psi_{i}(x)}{\partial x_{r}} \frac{\partial \psi_{j}(x)}{\partial x_{s}}\right|>0 \text {, and } K \text { is a constant by (2.1.2). }
$$


Then if $u \in W^{2, p}(Z) \cap W_{0}^{1, p}(Z)$ and $L_{v} u \in L^{p}(Z)$, with $1<p<\infty$, we have the estimate

$$
\|u\|_{W^{2, p}(Z)} \leq C\left(\left\|L_{v} u\right\|_{L^{P}(Z)}+\|u\|_{L^{p}(Z)}\right),
$$

where $C$ is constant (independent of $v$ ) dependent on $N, P, \lambda, \Lambda, \partial Z, Z, \psi$ and the moduli of continuity of the coefficients $a_{i j}(x, r)$ with respect to $x$ on $\bar{Z}$.

Proof. $\psi=\left(\psi_{1}, \ldots, \psi_{N}\right)$ is $C^{1,1}$ diffeomorphism from $\bar{Z}$ onto $\bar{B}$. Let $y=\psi(x)$ for $x \in Z, \tilde{u}(y)=u(x), \tilde{v}(y)=v(x)$ and $\tilde{L}_{\tilde{v}} \tilde{u}(y)=L_{v} u(x)$, where

$$
\begin{gathered}
\tilde{L}_{\tilde{v}} \tilde{u}(y)=\sum_{i, j=1}^{N} \tilde{a}_{i j}(y, \tilde{v}(y)) \frac{\partial^{2} \tilde{u}}{\partial y_{i} \partial y_{j}}+\sum_{i=1}^{N} \tilde{b}_{i}(y, \tilde{v}(y)) \frac{\partial \tilde{u}}{\partial y_{i}}+c(y, \tilde{v}(y)) \tilde{u} \quad \text { in } B \\
\tilde{a}_{i j}(y, \tilde{v}(y))=\sum_{r, s=1}^{N} \frac{\partial \psi_{i}}{\partial x_{r}} \frac{\partial \psi_{j}}{\partial x_{s}} a_{r s}(x, u(x)), \\
\tilde{b_{i}}(y, \tilde{v}(y))=\sum_{r, s=1}^{N} \frac{\partial^{2} \psi_{i}}{\partial x_{r} \partial x_{s}} a_{r s}+\sum_{r=1}^{N} \frac{\partial \psi_{i}}{\partial x_{r}} b_{r}(x, u(x)), \text { and } \tilde{c}(y, \tilde{v}(y))=c(x, u(x)) .
\end{gathered}
$$

It is readily seen that $\tilde{a}_{i j} \in C^{0,1}(\bar{B} \times \mathbb{R}), \tilde{b}_{i}, \tilde{c} \in L^{\infty}(B \times \mathbb{R})$. For all $\xi=$ $\left(\xi_{1}, \ldots, \xi_{N}\right) \in \mathbb{R}^{N}$, we have

$$
\begin{aligned}
\mathbf{1}^{\mathbf{0}} \quad \sum \tilde{a} \xi_{i} \xi_{j} & =\xi \tilde{a} \xi^{T} \\
& =(\xi G) a(\xi G)^{T} \\
& \geq \lambda|\xi G|^{2} \\
& =\lambda(\xi G)(\xi G)^{T} \\
& =\lambda \xi G G^{T} \xi^{T} \\
& \geq \lambda \alpha|\xi|^{2}=\tilde{\lambda}|\xi|^{2} \quad \text { by }(2.1 .7), \text { where } \tilde{\lambda}=\alpha \lambda,
\end{aligned}
$$

$\mathbf{2}^{\mathbf{0}} \quad y \in B: \quad \operatorname{osc} \tilde{a}(y, r)=\max _{1 \leq i, j \leq N} \operatorname{osc} \tilde{a}_{i j}(y, r)$

$$
\begin{aligned}
& \leq \max _{1 \leq i, j \leq N} \sum_{r, s}\left|\frac{\partial \psi_{i}(x)}{\partial x_{r}} \frac{\partial \psi_{j}(x)}{\partial x_{s}}\right| \text { osc } a_{r s}(x, r) \\
& \leq \beta \frac{\lambda}{4\left(\frac{\beta}{\alpha}\right) K}=\frac{\alpha \lambda}{4 K}=\frac{\tilde{\lambda}}{4 K} \quad \text { by (2.1.5), }
\end{aligned}
$$

$y \in \partial B: \quad$ osc $\tilde{a}(y, r) \leq \frac{\tilde{\lambda}}{8 N^{2} K} \quad$ by (2.1.6), 


$$
\begin{aligned}
3^{\mathbf{0}}\left|\tilde{a}_{i j}\right| & \leq \beta \Lambda \quad \forall i, j,|\tilde{c}| \leq \Lambda, \\
\left|\tilde{b}_{i}\right| & =\left|\sum_{r, s=1}^{N} \frac{\partial^{2} \psi_{i}}{\partial x_{r} \partial x_{s}} a_{r s}+\sum_{r=1}^{N} \frac{\partial \psi_{i}}{\partial x_{r}} b_{r}(x, u(x))\right| \\
& \leq \beta_{1} \Lambda \quad \forall i,
\end{aligned}
$$

where

$$
\max _{x \in \bar{Z}, 1 \leq i \leq N}\left|\sum_{r, s}^{N} \frac{\partial^{2} \psi_{i}}{\partial x_{r} \partial x_{s}}\right|+\left|\sum_{r, s}^{N} \frac{\partial \psi_{i}}{\partial x_{r}}\right|=\beta_{1} \text { for a constant } \beta_{1}>0
$$

Hence we get $\left|\tilde{a}_{i j}\right|,\left|\tilde{b}_{i}\right|,|\tilde{c}| \leq \tilde{\Lambda}=\max \left\{1, \beta_{1}, \beta\right\} \Lambda$, osc $\tilde{a}(y, r) \leq \frac{\tilde{\lambda}}{4 K} \forall y \in B$ and osc $\tilde{a}(y, r) \leq \frac{\tilde{\lambda}}{8 N^{2} K} \forall y \in \partial B$. Since the coefficient of $\tilde{L}$ satisfies the assumption of [3, Prop. 3.1.2], we have the global estimate of $W^{2, p}$ on $\tilde{u}$ by (2.1.3),

$$
\|\tilde{u}\|_{W^{2, p}(B)} \leq C\left(\|\tilde{u}\|_{L^{p}(B)}+\|\tilde{L} \tilde{v} \tilde{u}\|_{L^{P}(B)}\right),
$$

where $C=C(N, p, \tilde{\lambda}, \tilde{\Lambda}, \psi)$ and $C$ is independent of $v$. Since $G$ is a nonsingular bounded operator for all $x \in \bar{Z}$, we have

$$
\begin{aligned}
\int_{B}|\tilde{u}(y)|^{p} d y & =\int_{Z}|u(x)|^{p}|J \psi(x)| d x \\
& \leq \max _{x \in \bar{Z}}|\operatorname{det} G| \int_{Z}|u(x)|^{p} d x,
\end{aligned}
$$

where $J \psi(x)=\operatorname{det} G$, where implies that $\|\tilde{u}\|_{L^{p}(B)} \leq \sigma\|u\|_{L^{p}(Z)}$, where $\sigma=$ $\left(\max _{x \in \bar{Z}}|\operatorname{det} G|\right)^{1 / p}>0$. Similarly, we obtain

$$
\begin{gathered}
\left\|\tilde{L}_{\tilde{v} \tilde{u}}\right\|_{L^{P}(B)} \leq \sigma\left\|L_{v} u\right\|_{L^{P}(Z)}, \\
\int_{Z}|u(x)|^{p} d x=\int_{B}|\tilde{u}(y)|^{p}\left|J \psi^{-1}(y)\right| d y \\
\leq \max _{y \in \bar{B}}\left|J \psi^{-1}(y)\right| \int_{B}|\tilde{u}(u)|^{p} d y
\end{gathered}
$$

implies that $\|u\|_{L^{p}(Z)} \leq \rho\|\tilde{u}\|_{L^{p}(B)}$, where $\rho=\left(\min _{x \in \bar{Z}}|\operatorname{det} G|\right)^{-1 / p}>0$, 


$$
\begin{aligned}
& \left\|\frac{\partial u}{\partial x_{i}}\right\|_{L^{p}(Z)}=\left\|\sum_{r}^{N} \frac{\partial \tilde{u}}{\partial y_{r}} \frac{\partial y_{r}}{\partial x_{i}}\right\|_{L^{p}(Z)} \\
& \leq\left(\int_{Z}\left|\frac{\partial \tilde{u}(\psi(x))}{\partial y_{1}} \frac{\partial y_{1}}{\partial x_{i}}\right|^{p} d x\right)^{\frac{1}{p}}+\ldots+\left(\int_{Z}\left|\frac{\partial \tilde{u}(\psi(x))}{\partial y_{N}} \frac{\partial y_{N}}{\partial x_{i}}\right|^{p} d x\right)^{\frac{1}{p}} \\
& \leq \max _{x \in \bar{Z}, 1 \leq r \leq N}\left|\frac{\partial y_{r}}{\partial x_{i}}\right|\left[\left(\int_{Z}\left|\frac{\partial \tilde{u}(\psi(x))}{\partial y_{1}}\right|^{p} d x\right)^{\frac{1}{p}}+\ldots+\left(\int_{Z}\left|\frac{\partial \tilde{u}(\psi(x))}{\partial y_{N}}\right|^{p} d x\right)^{\frac{1}{p}}\right] \\
& \leq \max _{x \in \bar{Z}, 1 \leq r \leq N}\left|\frac{\partial y_{r}}{\partial x_{i}}\right|\left[\left(\int_{B}\left|\frac{\partial \tilde{u}(y)}{\partial y_{1}}\right|^{p}\left|J \psi^{-1}(y)\right| d y\right)^{\frac{1}{p}}+\ldots+\left(\int_{Z}\left|\frac{\partial \tilde{u}(y)}{\partial y_{N}}\right|^{p}\left|J \psi^{-1}(y)\right| d y\right)^{\frac{1}{p}}\right] \\
& \leq \max _{x \in \bar{Z}, 1 \leq r \leq N}\left|\frac{\partial y_{r}}{\partial x_{i}}\right| \rho \sum_{r}^{N}\left\|\frac{\partial \tilde{u}}{\partial y_{r}}\right\|_{L^{p}(B)} \\
& \leq \beta_{1} \rho \sum_{r}^{N}\left\|\frac{\partial \tilde{u}}{\partial y_{r}}\right\|_{L^{p}(B)} \text { by (2.1.9), }
\end{aligned}
$$

which implies that

$$
\left\|\frac{\partial u}{\partial x_{i}}\right\|_{L^{p}(Z)} \leq \beta_{1} \rho \sum_{r}^{N}\left\|\frac{\partial \tilde{u}}{\partial y_{r}}\right\|_{L^{p}(B)}
$$

for all $i$, and

$$
\begin{aligned}
\left\|\frac{\partial^{2} u}{\partial x_{i} \partial x_{j}}\right\|_{L^{p}(Z)}= & \left\|\sum_{r, s}^{N} \frac{\partial^{2} \tilde{u}}{\partial y_{r} \partial y_{s}} \frac{\partial y_{r}}{\partial x_{i}} \frac{\partial y_{s}}{\partial x_{j}}+\sum_{r}^{N} \frac{\partial \tilde{u}}{\partial y_{r}} \frac{\partial^{2} y_{r}}{\partial x_{i} \partial x_{j}}\right\|_{L^{p}(Z)} \| \\
\leq & \left\|\sum_{r, s}^{N} \frac{\partial^{2} \tilde{u}}{\partial y_{r} \partial y_{s}} \frac{\partial y_{r}}{\partial x_{i}} \frac{\partial y_{s}}{\partial x_{j}}\right\|_{L^{p}(Z)}+\left\|\sum_{r}^{N} \frac{\partial \tilde{u}}{\partial y_{r}} \frac{\partial^{2} y_{r}}{\partial x_{i} \partial x_{j}}\right\|_{L^{p}(Z)}\left|\frac{\partial y_{r}}{\partial x_{i}} \frac{\partial y_{s}}{\partial x_{j}}\right| \sum_{r, s}^{N}\left\|\frac{\partial^{2} \tilde{u}}{\partial y_{r} \partial y_{s}}\right\|_{L^{p}(Z)} \\
& +\max _{x \in \bar{Z}, 1 \leq r \leq N}\left|\frac{\partial^{2} y_{r}}{\partial x_{i} \partial x_{j}}\right| \sum_{r, s}^{N}\left\|\frac{\partial \tilde{u}}{\partial y_{r}}\right\|_{L^{p}(Z)} \\
\leq & \beta \rho \sum_{r, s}^{N}\left\|\frac{\partial^{2} \tilde{u}}{\partial y_{r} \partial y_{s}}\right\|_{L^{p}(B)}+\beta_{1} \rho \sum_{r, s}^{N}\left\|\frac{\partial \tilde{u}}{\partial y_{r}}\right\|_{L^{p}(B)}
\end{aligned}
$$

which implies that

$$
\left\|\frac{\partial^{2} u}{\partial x_{i} \partial x_{j}}\right\|_{L^{p}(Z)} \leq \beta \rho \sum_{r, s}^{N}\left\|\frac{\partial^{2} \tilde{u}}{\partial y_{r} \partial y_{s}}\right\|_{L^{p}(B)}+\beta_{1} \rho \sum_{r, s}^{N}\left\|\frac{\partial \tilde{u}}{\partial y_{r}}\right\|_{L^{p}(B)}
$$


for all $i$ and $j$. To summarize, we can obtain that

$$
\|u\|_{W^{2, p}(Z)} \leq \eta\|\tilde{u}\|_{W^{2, p}(B)},
$$

where $\eta$ is a nonnegative constant dependent of $\psi$. Thus, returning to our original coordinate $Z$, we have got our estimates,

$$
\|u\|_{W^{2, p}(Z)} \leq C\left(\|u\|_{L^{p}(Z)}+\left\|L_{v} u\right\|_{L^{P}(Z)}\right),
$$

where $C=C(N, p, \lambda, \Lambda, Z, \partial Z, \psi)$.

\subsection{Existence Results}

The results of the preceding section will now be applied to establish the existence of solutions of the following quasilinear elliptic problem:

$$
\begin{cases}L u=\sum_{i, j=1}^{N} a_{i j}(x, u) \frac{\partial^{2} u}{\partial x_{i} \partial x_{j}}+\sum_{i=1}^{N} b_{i}(x, u) \frac{\partial u}{\partial x_{i}}+c(x, u) u=f(x) & \text { in } Z \\ u=0 & \text { on } \partial Z\end{cases}
$$

where $f \in L^{p}(Z), p \geq N$. For the moment, we suppose $a_{i j} \in C^{0,1}(\bar{Z} \times \mathbb{R})$, $a_{i j}, \partial a_{i j} / \partial x_{i}, \partial a_{i j} / \partial r, b_{i}, c$ are bounded Carathédory functions, with $c \leq 0$. By the existence and uniqueness theorem of the strong solution for the Dirichlet problem [1, p. 241], there exists a unique solution $u \in W^{2, p}(Z) \cap W_{0}^{1, p}(Z)$ to the equation $L_{v} u=f(x)$ for each $v \in W_{0}^{1, p}$. Consider the mapping $\mathrm{F}$ which assigns $v \in W^{2, p}(Z) \cap W_{0}^{1, p}(Z)$ to the solution $u \in W^{2, p}(Z) \cap W_{0}^{1, p}(Z)$ satisfying the following equation

$$
L_{v} u=\sum_{i, j=1}^{N} a_{i j}(x, v) \frac{\partial^{2} u}{\partial x_{i} \partial x_{j}}+\sum_{i=1}^{N} b_{i}(x, v) \frac{\partial u}{\partial x_{i}}+c(x, v) u=f(x) \quad x \in Z,
$$

i.e., $F: v \in W^{2, p}(Z) \cap W_{0}^{1, p}(Z) \mapsto F(v)=u \in W^{2, p}(Z) \cap W_{0}^{1, p}(Z)(F$ is well-defined provided $p>N / 2$ ). From the following theorem, we can obtain the $L^{\infty}$ estimate for the solution $u=F(v)$ to equation (2.2.2).

Weak Maximum Principle of A. D. Aleksandrov [1, p. 220]:

Consider

$$
L u=\sum_{i, j=1}^{N} a_{i j}(x) \frac{\partial^{2} u}{\partial x_{i} \partial x_{j}}+\sum_{i=1}^{N} b_{i}(x) \frac{\partial u}{\partial x_{i}}+c(x) u=f(x),
$$

where $L$ is elliptic in the domain $\Omega$, and the coefficient matrix $A=\left[a_{i j}\right]$ is positive definite everywhere in $\Omega$. For such operators, we will let $D$ denote the determinant of $A$ and set $D^{*}=D^{1 / n}$ so that $D^{*}$ is the geometric mean of the eigenvalues of $A$ such 
that $0<\omega \leq D^{*} \leq \gamma$, where $\omega$ and $\gamma$ are the minimum and maximum eigenvalues of $A$ respectively. If $|b| / D^{*}, f / D^{*} \in L^{N}(\Omega), c \leq 0$ in $\Omega, u \in C^{0}(\bar{\Omega}) \cap W_{\text {loc }}^{2, N}(\Omega)$, and $L u \geq f$ in bounded domain $\Omega$, then

$$
\sup _{\Omega} u \leq \sup _{\partial \Omega} u^{+}+C\left\|\frac{f}{D^{*}}\right\|_{L^{N}(\Omega)},
$$

where $C$ is a constant dependent on $N$, $\operatorname{diam} \Omega$, and $\left\|b / D^{*}\right\|_{L^{N}(\Omega)}$.

For the equation (2.2.2), $u$ is zero on the boundary of $Z$. Since $a_{i j}$ is bounded, $D^{*}=D^{1 / N}$ is a bounded function and $0<\lambda \leq D^{*}$, where $\lambda$ is an ellipticity constant in (2.1.1). For $p \geq N$, we then have $f \in L^{N}(Z)$ and

$$
\sup _{Z}|u| \leq C\|f\|_{L^{N}(Z)},
$$

where $C$ is a constant dependent on $N, \lambda, \Lambda$, and diam $Z$ (the maximum principle is valid for $p \geq N)$. With the aid of (2.1.8), we have the following inequality

$$
\|u\|_{W^{2, p}} \leq C\|f\|_{L^{p}(Z)} \quad \text { for all } u=F(v), v \in W^{2, p}(Z) \cap W_{0}^{1, p}(Z) .
$$

We proceed to show that there exists a fixed point $u$ of $F$; $u$ then is a solution of the problem (2.2.1) by the Schauder Fixed Point Theorem. It suffices to show that $F: \mathcal{K} \rightarrow \mathcal{K}$ is continuous and $\mathcal{K}$ is a compact convex set in a Banach space. We have the following lemma.

Lemma 2.2.1. Let $p \geq N$. Under the hypotheses of Proposition 2.1.1, the mapping $F: W^{2, p}(Z) \cap W_{0}^{1, p}(Z) \rightarrow W^{2, p}(Z) \cap W_{0}^{1, p}(Z)$ is continuous in the topology of $W^{1, p}(Z)$.

Proof: If $\left\{v_{n}\right\} \subset W^{2, p}(Z) \cap W_{0}^{1, p}(Z)$ and $v_{n} \rightarrow v$ in $W^{1, p}(Z)$, then there exists a subsequence, denoted by $v_{n}$, such that $v_{n} \rightarrow v$ a.e., and $\nabla v_{n} \rightarrow \nabla v$ a.e. Let $u_{n}=F\left(v_{n}\right)$ and $u=F(v)$. We will show that $u_{n} \rightarrow u$ in $W^{1, p}(Z)$. Since $f \in L^{p}(Z)$, and $p \geq N$, by (2.2.4), $\left\{u_{n}\right\}$ is bounded in $W^{2, p}(Z)$. Also since $W^{2, p}(Z) \hookrightarrow W^{1, p}(\bar{Z})$ is a compact imbedding, there exists a subsequence (we relabel as $\left\{u_{n}\right\}$ ) such that $u_{n} \rightarrow w$ in $W^{1, p}(Z)$ with $w \in W^{1, p}(Z), u_{n} \rightarrow w$ a.e., and $\nabla u_{n} \rightarrow \nabla w$ a.e. We claim that $w$ is a weak solution of the following equation

$$
\sum_{i, j=1}^{N} a_{i j}(v) \frac{\partial^{2} w}{\partial x_{i} \partial x_{j}}+\sum_{i=1}^{N} b_{i}(x, v) \frac{\partial w}{\partial x_{i}}+c(x, v) w=f(x) .
$$

It suffies to show that

$$
\begin{aligned}
\int_{Z} \sum_{i, j=1}^{N} a_{i j}(v) \frac{\partial w}{\partial x_{j}} \frac{\partial \phi}{\partial x_{i}} & +\int_{Z} \sum_{i=1}^{N}\left[\sum_{j=1}^{N}\left(\frac{\partial a_{j i}(v)}{\partial x_{j}}+\frac{\partial a_{j i}(v)}{\partial r} \frac{\partial v}{\partial x_{j}}\right)-b_{i}(v)\right] \frac{\partial w}{\partial x_{i}} \phi \\
+\int_{Z}(-c(x, v)) w \phi & =\int_{Z}-f \phi \quad \text { for all } \phi \in C_{0}^{\infty}(Z) .
\end{aligned}
$$


Let $\phi \in C_{0}^{\infty}(Z)$. Since $u_{n}=F\left(v_{n}\right)$, we have

$$
\begin{aligned}
\int_{Z} \sum_{i, j=1}^{N} a_{i j}\left(v_{n}\right) \frac{\partial u_{n}}{\partial x_{j}} \frac{\partial \phi}{\partial x_{i}} & +\int_{Z} \sum_{i=1}^{N}\left[\sum_{j=1}^{N}\left(\frac{\partial a_{j i}\left(v_{n}\right)}{\partial x_{j}}+\frac{\partial a_{j i}\left(v_{n}\right)}{\partial r} \frac{\partial v_{n}}{\partial x_{j}}\right)-b_{i}\left(v_{n}\right)\right] \frac{\partial u_{n}}{\partial x_{i}} \phi \\
& +\int_{Z}\left(-c\left(v_{n}\right)\right) u_{n} \phi=\int_{Z}-f \phi .
\end{aligned}
$$

Since $a_{i j}, \partial a_{i j} / \partial x_{i}, \partial a_{i j} / \partial r, b_{i}, c$ are bounded Carathédory functions, $u_{n} \rightarrow w$ a.e., $\nabla u_{n} \rightarrow \nabla w$ a.e., by Lebesgue's Dominated Convergence Theorem we have

$$
\begin{aligned}
\int_{Z} \sum_{i, j=1}^{N} a_{i j}\left(v_{n}\right) \frac{\partial u_{n}}{\partial x_{j}} \frac{\partial \phi}{\partial x_{i}} & +\int_{Z} \sum_{i=1}^{N}\left[\sum_{j=1}^{N}\left(\frac{\partial a_{j i}\left(v_{n}\right)}{\partial x_{j}}+\frac{\partial a_{j i}\left(v_{n}\right)}{\partial r} \frac{\partial v_{n}}{\partial x_{j}}\right)-b_{i}\left(v_{n}\right)\right] \frac{\partial u_{n}}{\partial x_{i}} \phi \\
& +\int_{Z}(-c(v)) w \phi=\int_{Z}(-f) \phi \quad \text { for all } \phi \in C_{0}^{\infty}(Z) .
\end{aligned}
$$

Hence (2.2.5) holds. It follows from the uniqueness of the solution to equation (2.2.2) that we have $u=w$ and $u_{n} \rightarrow u$ in $W^{1, p}(Z)$. Therefore, the proof is completed.

Proposition 2.2.2. Let $Z$ be a bounded smooth domain in $\mathbb{R}^{N}$ satisfying the assumption of Proposition 2.1.1. Suppose $a_{i j} \in C^{0,1}(\bar{Z} \times \mathbb{R}), a_{i j}, \partial a_{i j} / \partial x_{i}$, $\partial a_{i j} / \partial r, b_{i}, c \in L^{\infty}(Z \times \mathbb{R}), c \leq 0$ with $i, j=1, \ldots N$. Then, for $p \geq N$, there exist a solution $u \in W^{2, p}(Z) \cap W_{0}^{1, p}(Z)$ to problem (2.2.1).

Proof. Consider $u=F(v)$ for $v \in W^{2, p}(Z) \cap W_{0}^{1, p}(Z)$. According to (2.2.4), we can obtain a nonnegative constant $K$, such that

$$
\|u\|_{W^{2, p}(Z)} \leq K \quad \text { for } v \in W^{2, p}(Z) \cap W_{0}^{1, p}(Z) .
$$

Let

$$
\mathcal{K}=\left\{v \in W^{2, p}(Z) \cap W_{0}^{1, p}(Z) \mid \quad\|v\|_{W^{2, p}(Z)} \leq K\right\} .
$$

Then $F$ is continuous from $\mathcal{K}$ into itself in the topology of $W^{1, p}$ by Lemma 2.2.1. Since $\mathcal{K}$ is bounded in $W^{2, p}(Z)$ and $W^{2, p} \hookrightarrow W^{1, p}$ is a compact imbedding, $\mathcal{K}$ is a precompact set in $W^{1, p}(Z)$. We claim that $\mathcal{K}$ is closed in $W^{1, p}(Z)$. To see this, let $\left\{u_{n}\right\} \subset \mathcal{K}$ be such that $u_{n} \rightarrow u$ in $W^{1, p}(Z)$. Since $\left\{u_{n}\right\}$ is bounded in $W^{2, p}$ and $W^{2, p}$ is a reflexive space, there exists a subsequence weakly convergent to $w \in$ $W^{2, p}$. It can be shown that $w=u$. With the aid of $\|u\|_{W^{2, p}} \leq \underline{\lim }_{n}\left\|u_{n}\right\|_{W^{2, p}} \leq K$, we obtain that $\mathcal{K}$ is closed in $W^{1, p}$. Hence $\mathcal{K}$ is a compact and convex set in $W^{1, p}$ which is a Banach space. It follows readily from the Schauder Fixed Point Theorem that there exists a solution $u \in W^{2, p}(Z) \cap W_{0}^{1, p}(Z)$ of problem (2.2.1) in $\mathcal{K}$.

Remark 2.2.3. It follows from the proof of Proposition 2.2.2 that the solutions of equation (2.2.1) are bounded in $W^{2, p}(Z)$. 


\section{An Application to the Existence of Strong Solutions to ome Quasilinear Elliptic Problems}

In this section, we consider the following quasilinear elliptic problem:

$$
\begin{cases}\sum_{i, j=1}^{N} a_{i j}(x, u) \frac{\partial^{2} u}{\partial x_{i} \partial x_{j}}+\sum_{i=1}^{N} b_{i}(x, u) \frac{\partial u}{\partial x_{i}}+c(x, u) u=f(x, u, \nabla u) & \text { in } Z \\ u=0 & \text { on } \partial Z\end{cases}
$$

where $Z$ is a smooth domain in $\mathbb{R}^{N}, a_{i j} \in C^{0,1}(\bar{Z} \times \mathbb{R}), a_{i j}, \partial a_{i j} / \partial x_{i}, \partial a_{i j} / \partial r, b_{i}$, $c, f(x, r, \xi)$ are Carathéodory functions and $\sum_{i, j=1}^{N} a_{i j} \xi_{i} \xi_{j} \geq \lambda|\xi|^{2}$ with a nonnegative constant $\lambda$. The results of Section 2 are used to prove the following theorem.

Theorem 3.1. Let $Z$ be a bounded smooth domain in $\mathbb{R}^{N}$ satisfying the assumption of Proposition 2.1.2. Suppose $a_{i j} \in C^{0,1}(\bar{Z} \times \mathbb{R}), a_{i j}$, $\partial a_{i j} / \partial x_{i}, \partial a_{i j} / \partial r$, $b_{i}, c \in L^{\infty}(Z \times \mathbb{R})$ with $i, j=1, \ldots N,-c \geq \alpha_{0}>0$ for some constant $\alpha_{0}$ and

$$
|f(x, r, \xi)| \leq C_{0}+h(|r|)|\xi|^{\theta} \quad 0 \leq \theta<2,
$$

where $C_{0}$ is a nonnegative constant and $h(|r|)$ is a locally bounded function. Then there exists a solution $u \in W^{2, p}(Z) \cap W_{0}^{1, p}(Z)$ to problem (3.1).

The proof of Theorem 3.1 is done in the following steps:

(1) Approach equation (3.1) by truncation, and then prove the existence of approximating solutions $\left\{u_{n}\right\}$.

(2) Establish $L^{\infty}$ bound for the subsequence of $\left\{u_{n}\right\}$.

(3) Establish $W^{2, p}$ bound for the subsequence of $\left\{u_{n}\right\}$.

(4) Pass the approximating problem to the limit.

(5) Verify that the limit $u$ of the subsequence of approximating solutions $\left\{u_{n}\right\}$ in $W_{0}^{1, p}$ belongs to $W^{2, p} \cap W_{0}^{1, p}$.

Lemma 3.2. Suppose that $f(x, r, \xi)$ has an $L^{\infty}$ bound. Then for $1 \leq p<$ $\infty$ there exists a solution $u \in W^{2, p}(Z) \cap W_{0}^{1, p}(Z)$ to problem (3.1) under the assumption of Theorem 3.1.

Proof: For each $v \in W^{1, p}(Z), f(x, v, \nabla v) \in L^{\infty}(Z) \subset L^{p}(Z)$, the existence and uniqueness theorem [1, p. 241] asserts that there exists a unique $u \in W^{2, p}(Z) \cap$ $W_{0}^{1, p}(Z)$ to the equation

$$
L_{v} u=\sum_{i, j=1}^{N} a_{i j}(x, v) \frac{\partial^{2} u}{\partial x_{i} \partial x_{j}}+\sum_{i=1}^{N} b_{i}(x, v) \frac{\partial u}{\partial x_{i}}+c(x, v) u=f(x, v, \nabla v) .
$$


Moreover, by Proposition 2.1.1, we have the global estimate

$$
\|u\|_{W^{2, p}(Z)} \leq C\left(\|u\|_{L^{p}(Z)}+\|f(x, v, \nabla v)\|_{L^{p}(Z)}\right),
$$

with a constant $C>0$ (independent of $v$ ). Without loss of generality, we assume $p \geq$ $N$. Since $f \in L^{\infty}$, from the Maximum Principle of A. D. Aleksandrov [1, p. 220], we obtain $\|u\|_{W^{2, p}(Z)} \leq M$ for some constant $M>0$. Following the same process in subsection 2.2, let $T$ be the map which associates $v \in W^{2, p}(Z) \cap W_{0}^{1, p}(Z)$ to the solution $u \in W^{2, p}(Z) \cap W_{0}^{1, p}(Z)$ satisfying $L_{v} u=f(x, v, \nabla v)$. Notice that since $f(x, r, \xi)$ is a bounded Carathédory function, we have $f\left(x, v_{n}, \nabla v_{n}\right) \rightarrow f(x, v, \nabla v)$ in $L^{1}(Z)$ if $v_{n} \rightarrow v$ in $W^{1, p}$ and $v_{n} \rightarrow v, \nabla v_{n} \rightarrow \nabla v$ a.e. By a similar argument as in the proof of Lemma 2.2.1, we can show that $T: W^{2, p}(Z) \cap W_{0}^{1, p}(Z) \rightarrow$ $W^{2, p}(Z) \cap W_{0}^{1, p}(Z)$ is continuous in the topology $W^{1, p}(Z)$. The existence of the solution $u \in W^{2, p}(Z) \cap W_{0}^{1, p}(Z)$ to problem (3.1) then follows from Proposition 2.2.2.

Let's now consider the approximating problem of problem (3.1):

(3.3) $\begin{cases}\sum_{i, j=1}^{N} a_{i j}(x, u) \frac{\partial^{2} u}{\partial x_{i} \partial x_{j}}+\sum_{i=1}^{N} b_{i}(x, u) \frac{\partial u}{\partial x_{i}}+c(x, u) u=f_{n}(x, u, \nabla u) & \text { in } Z, \\ u=0 & \text { on } \partial Z\end{cases}$

where $f_{n}(x, r, \xi)$ is the truncation of $f$ by $\pm n$, i.e.,

$$
f_{n}(x, r, \xi)=\left\{\begin{aligned}
n & \text { if } f(x, r, \xi) \geq n \\
f(x, r, \xi) & \text { if }|f(x, r, \xi)| \leq n \\
-n & \text { if } f(x, r, \xi) \leq-n
\end{aligned}\right.
$$

Clearly, $f_{n}(x, r, \xi) \in L^{\infty}(Z) \subset L^{p}(Z)$ for all $n$. According to Lemma 3.2, for each $1 \leq p<\infty$, there exists a solution $u_{n} \in W^{2, p}(Z) \cap W_{0}^{1, p}(Z)$ to the approximating problem (3.3). Without loss of generality, we assume $p>N \geq 3$.

Lemma 3.3. Under the assumption of Theorem 3.1, there exists a subsequence of the approximating solution $\left\{u_{n}\right\}$ to problem (3.1) which is $L^{\infty}$ bounded.

Proof: Since $a_{i j} \in C^{0,1}(\bar{Z} \times \mathbb{R})$, the problem in (3.1) can be written in the following divergence form:

$$
-\sum_{i, j=1}^{N} \frac{\partial}{\partial x_{i}} a_{i j}(x, u) \frac{\partial u}{\partial x_{j}}+\tilde{f}(x, u, \nabla u)=0,
$$


where

$$
\begin{aligned}
\tilde{f}(x, u, \nabla u)= & \sum_{i=1}^{N}\left\{\sum_{j=1}^{N}\left[\frac{\partial a_{j i}(x, u)}{\partial x_{j}}+\frac{\partial a_{j i}(x, u)}{\partial r} \frac{\partial u}{\partial x_{j}}\right]-b_{i}(x, u)\right\} \frac{\partial u}{\partial x_{i}} \\
& -c(x, u) u+f(x, u, \nabla u) .
\end{aligned}
$$

Since $a_{i j}, \partial a_{i j} / \partial x_{i}, \partial a_{i j} / \partial r, b_{i}, c \in L^{\infty}(Z \times \mathbb{R})$ with $i, j=1, \ldots N$, there exists a constant $\Lambda>0$ such that $a_{i j}, \partial a_{i j} / \partial x_{i}, \partial a_{i j} / \partial r, b_{i}, c \leq \Lambda$. Thus,

$$
\begin{aligned}
& \left|\sum_{i, j=1}^{N}\left[\frac{\partial a_{j i}(x, u)}{\partial x_{j}} \frac{\partial u}{\partial x_{i}}+\frac{\partial a_{j i}(x, u)}{\partial r} \frac{\partial u}{\partial x_{i}} \frac{\partial u}{\partial x_{j}}\right]-\sum_{i=1}^{N} b_{i}(x, u) \frac{\partial u}{\partial x_{i}}-c(x, u) u\right| \\
\leq & \frac{\Lambda N}{2}\left(N+|\nabla u|^{2}\right)+\Lambda\left(|\nabla u|^{2}\right)+\frac{\Lambda}{2}\left(N+|\nabla u|^{2}\right)+\Lambda|u| \\
\leq & M|\nabla u|^{2}+\Lambda|u|+C^{\prime},
\end{aligned}
$$

for some nonnegative constants $M=(\Lambda / 2)(N+3 / 2), C^{\prime}=(\Lambda N / 2)(N+1)$. Together with the hypothesis of (3.2), we have

$$
\begin{aligned}
|\tilde{f}(x, r, \xi)| & \leq C_{0}+h(|r|)\left(1+|\xi|^{2}\right)+M|\xi|^{2}+\Lambda|r|+C^{\prime} \\
& \leq b(|r|)\left(1+|\xi|^{2}\right)
\end{aligned}
$$

where $b$ is an increasing function from $\mathbb{R}^{+}$into $\mathbb{R}^{+}$. Let $\phi=-C_{0} / \alpha_{0}$ and $\psi=$ $C_{0} / \alpha_{0}$. It's clear that $\phi$ and $\psi$ are the sub- and supper-solution of problem (3.1), respectively. Thus, it follows from [5, Proposition 3.6] that there is a subsequence of the approximating sequence of solutions $\left\{u_{n}\right\}$ to problem (3.1) ( we relabel as $\left.\left(u_{n}\right)\right)$ with $\phi \leq u_{n} \leq \psi$ in $Z$. Hence $\left(u_{n}\right)$ are $L^{\infty}(Z)$ bounded.

Theorem (Interpolation Inequality of Gagliardo-Nirenberg).

Let $\Omega \subset \mathbb{R}^{N}$ be an open bounded regular set and $u \in L^{r} \cap W^{2, p}(\Omega)$ with $1 \leq p \leq \infty$ and $1 \leq r \leq \infty$. Then $u \in W^{1, q}(\Omega)$ where $q$ is the harmonic average of $p$ and $r$, that is $1 / q=((1 / 2)+(1 / p)) / 2$ and

$$
\|\nabla u\|_{L^{q}} \leq C\|u\|_{W^{2, p}}^{\frac{1}{2}}\|u\|_{L^{r}}^{\frac{1}{2}} .
$$

In particular $r=\infty$ and then $q=2 p$. We have $u \in W^{1,2 p}(\Omega)$ and

$$
\|\nabla u\|_{L^{2 p}} \leq C\|u\|_{W^{2, p}}^{\frac{1}{2}}\|u\|_{L^{\infty}}^{\frac{1}{2}} .
$$

Lemma 3.4. Under the assumptions of Theorem 3.1, there exists a subsequence of the approximating solution $\left\{u_{n}\right\}$ in $W^{2, p}(Z) \cap W_{0}^{1, p}(Z)$ to problem (3.1) which is $W^{2, p}$ bounded. 
Proof. By Lemma 3.3, there exists a sequence $\left\{u_{n}\right\}$ which is $L^{\infty}$ bounded. Since $h(|r|)$ is locally bounded, we have $\left|h\left(u_{n}\right)\right| \leq M$ for some constant $M>0$. According to (3.2), we have $\left|f_{n}\left(x, u_{n}, \nabla u_{n}\right)\right| \leq C_{0}+h\left(\left|u_{n}\right|\right)\left|\nabla u_{n}\right|^{\theta}, 0 \leq \theta<2$. Hence there exists a constant $C_{1}>0$ such that

$$
\left|f_{n}(x, r, \xi)\right| \leq C_{1}\left(1+\left|\nabla u_{n}\right|^{\theta}\right) .
$$

Since $\theta<2$, there exists a constant $C_{\epsilon}>0$ for all $\epsilon>0$ such that

$$
\left|\nabla u_{n}\right|^{\theta} \leq C_{\epsilon}+\epsilon\left|\nabla u_{n}\right|^{2} \text {. }
$$

Thus $\left|f_{n}\left(x, u_{n}, \nabla u_{n}\right)\right| \leq M_{1}+\epsilon C_{1}\left|\nabla u_{n}\right|^{2}$ for a constant $M_{1}>0$. With the help of the global estimate (2.1.9), we have

$$
\begin{aligned}
\left\|u_{n}\right\|_{W^{2, p}(Z)} & \leq C\left(\left\|u_{n}\right\|_{L^{p}}+\left\|f_{n}\left(x, u_{n}, \nabla u_{n}\right)\right\|_{L^{p}}\right) \\
& \leq M_{2}+\epsilon C_{2}\left\|\nabla u_{n}\right\|_{L^{2 P}}^{2}
\end{aligned}
$$

for some constant $M_{2}, C_{2}>0$. Since $u_{n} \in L^{\infty}(Z) \cap W^{2, p}(Z)$, from the interpolation of Gagliardo-Nirenberg Theorem, we obtain

$$
\begin{aligned}
\left\|u_{n}\right\|_{W^{2, p}(Z)} & \leq M_{2}+\epsilon C_{2}\left\|u_{n}\right\|_{W^{2, P}(Z)}\left\|u_{n}\right\|_{L^{\infty}(Z)} \\
& \leq M_{2}+\epsilon C_{3}\left\|u_{n}\right\|_{W^{2, P}(Z)},
\end{aligned}
$$

where $M_{2}, C_{3}$ are nonnegative constants. Hence, by choosing $C_{3} \epsilon=1 / 2$, we obtain $\left\|u_{n}\right\|_{W^{2, p}(Z)} \leq M_{3}$ for some constant $M_{3}>0$. Therefore, $\left\{u_{n}\right\}$ are $W^{2, p}$ bounded.

By Lemma 3.4, we get a sequence of approximating solutions to problem (3.1) which is $W^{2, p}$ bounded. It follows from the compactness of the imbedding $W^{2, p} \hookrightarrow W^{1, p}$ that there exists a norm convergent subsequence in $W^{1, p}$. We extract a subsequence, which is denoted again by $\left\{u_{n}\right\}$ such that

$$
u_{n} \rightarrow u \text { a.e., } \nabla u_{n} \rightarrow \nabla u \text { a.e., and } u_{n} \rightarrow u \text { in } W^{1, p} \text {. }
$$

In what follows, we show that $u$ is a solution of problem (3.1). By passing to the limit, we obtain

$$
\begin{aligned}
& \int \sum_{i, j=1}^{N} a_{i j}\left(u_{n}\right) \frac{\partial u_{n}}{\partial x_{j}} \frac{\partial \phi}{\partial x_{i}}+\int \sum_{i, j=1}^{N}\left[\frac{\partial a_{j i}\left(u_{n}\right)}{\partial x_{j}}+\frac{\partial a_{j i}\left(u_{n}\right)}{\partial r} \frac{\partial u_{n}}{\partial x_{j}}\right] \frac{\partial u_{n}}{\partial x_{i}} \phi \\
& -\int \sum_{i=1}^{N} b_{i}\left(u_{n}\right) \frac{\partial u_{n}}{\partial x_{i}} \phi-\int c\left(u_{n}\right) u_{n} \phi=\int-f_{n}\left(x, u_{n}, \nabla u_{n}\right) \phi \\
\rightarrow & \int \sum_{i, j=1}^{N} a_{i j}(u) \frac{\partial u}{\partial x_{j}} \frac{\partial \phi}{\partial x_{i}}+\int \sum_{i, j=1}^{N}\left[\frac{\partial a_{j i}(u)}{\partial x_{j}}+\frac{\partial a_{j i}(u)}{\partial r} \frac{\partial u}{\partial x_{j}}\right] \frac{\partial u}{\partial x_{i}} \phi \\
& -\int \sum_{i=1}^{N} b_{i}(u) \frac{\partial u}{\partial x_{i}} \phi-\int c(u) u \phi \quad \forall \phi \in C_{0}^{\infty}(Z) .
\end{aligned}
$$


The next lemma shows that $f_{n}\left(x, u_{n}, \nabla u_{n}\right) \rightarrow f(x, u, \nabla u)$ in $L^{1}(Z)$. Therefore, $u$ is a $W^{1, p}(Z)$ solution to the problem (3.1).

Theorem (Vitali Convergence Theorem).

Let $1 \leq p \leq \infty$ and $(\Omega, \Sigma, \mu)$ be a measurable space. Let $\left\{f_{n}\right\}$ be a sequence of functions in $L^{p}$ converging almost everywhere to a function $f$. Then $f$ is in $L^{p}$ and $\left\|f_{n}-f\right\|_{p}$ converges to zero if and only if

(1) $\lim _{\mu(E) \rightarrow 0} \int_{E}\left|f_{n}\right|^{p} d \mu=0$ uniformly $\forall n$;

(2) for each $\epsilon>0$ there exists a set $E_{\epsilon}$ such that $\mu\left(E_{\epsilon}\right)<\infty$ and $\int_{\Omega-E_{\epsilon}}\left|f_{n}\right|^{p} d \mu<$ $\epsilon$ for $n=1,2, \ldots$.

Lemma 3.5. $f_{n}\left(x, u_{n}, \nabla u_{n}\right) \rightarrow f(x, u, \nabla u)$ in $L^{1}(Z)$.

Proof. Since $f$ is a Carathédory function, $u_{n} \rightarrow u$ a.e., and $\nabla u_{n} \rightarrow \nabla u$ a.e. we have $f_{n}\left(x, u_{n}, \nabla u_{n}\right) \rightarrow f(x, u, \nabla u)$ a.e. According to (3.7), we have

$$
\begin{aligned}
\left|f_{n}\left(x, u_{n}, \nabla u_{n}\right)\right| & \leq C_{1}\left(1+\left|\nabla u_{n}\right|^{\theta}\right) \\
& \leq C_{1}\left(2+\left|\nabla u_{n}\right|^{2}\right) .
\end{aligned}
$$

Since $\left\{u_{n}\right\}$ is $H^{1}$ bounded with $p>N \geq 3,\left\{f_{n}\right\}$ is a sequence of functions in $L^{1}(Z)$. Now, by Vitali Convergence Theorem, we conclude that $f_{n}\left(x, u_{n}, \nabla u_{n}\right) \rightarrow$ $f(x, u, \nabla u)$ in $L^{1}(Z)$.

Lemma 3.6. Under the assumptions of Theorem 3.1, the limit $u$ of the approximating solutions $\left\{u_{n}\right\}$ to problem (3.1) belongs to $W^{2, p}(Z) \cap W_{0}^{1, p}(Z)$.

Proof. By Lemma 3.4, there exists a constant $M>0$ such that $\left\|u_{n}\right\|_{W^{2, p}(Z)} \leq$ $M$ for all $n$. Let

$$
\mathcal{K}=\left\{v \in W^{2, p}(Z) \cap W_{0}^{1, p}(Z) \mid \quad\|v\|_{W^{2, p}(Z)} \leq M\right\} .
$$

By the same argument as in the proof of Proposition 2.2.2, it follows that $\mathcal{K}$ is closed in $W^{1, p}$. Thus the limit $u$ of $\left(u_{n}\right)$ belongs to $W^{2, p}(Z) \cap W_{0}^{1, p}(Z)$.

Therefore, the existence of solutions in $W^{2, p}(Z) \cap W_{0}^{1, p}(Z)$ asserted in Theorem 3.1 now follows readily from Lemmas 3.2-3.6.

Lemma 3.7. If $f(x, r, \xi)$ has a quadratic growth in $\xi$, that is $\theta=2$ in (3.2), then there exists an $H^{1}$ bound for the approximating solutions $\left\{u_{n}\right\}$ to problem (3.1). 
Proof. The differential equation in (3.1) can be written in the following divergence form:

$$
-\sum_{i, j=1}^{N} \frac{\partial}{\partial x_{i}} a_{i j}(x, u) \frac{\partial u}{\partial x_{j}}-c(x, u) u=g(x, u, \nabla u)
$$

where

$$
g(x, u, \nabla u)=-f(x, u, \nabla u)+\sum_{i=1}^{N} b_{i}(u) \frac{\partial u}{\partial x_{i}}-\sum_{i, j=1}^{N} \frac{\partial a_{i j}}{\partial x_{i}} \frac{\partial u}{\partial x_{j}}-\sum_{i, j=1}^{N} \frac{\partial a_{i j}}{\partial r} \frac{\partial u}{\partial x_{i}} \frac{\partial u}{\partial x_{j}} .
$$

Since $f(x, r, \xi)$ satisfies (3.2), we have

$$
|g(x, r, \xi)| \leq C+E(|r|)|\xi|^{2},
$$

where $C$ is a nonnegative constant and $E$ is a locally bounded function in $\mathbb{R}^{+}$. Following from the proof of [6, Theorem 2.1], the approximating solution $\left\{u_{n}\right\}$ is $H^{1}$ bounded.

\section{REFERENCES}

1. D. Gilbarg and N. S. Trudinger, Elliptic Partial Differential Equations of Second Order, section edition, New York, Springer-Verlag, 1983.

2. S. Agmon, A. Douglis and L. Nirenberg, Estimates near the boundary for solutions of elliptic partial differential equations satisfying general boundary conditions I, Comm. Pure Appl. Math. 12 (1959), 623-727.

3. Chiung-Chiou Tsai and Tsang-Hai Kuo, On the Existence of Solutions to Some Quasilinear Elliptic Problems, Ph.D Thesis, National Chiao Tung University, Hsinchu, Taiwan, 1997.

4. Roger A. Horn and Charles R. Johnson, Matrix Analysis, Cambridge University Press, Cambridge, 1985.

5. L. Boccardo, F. Murat and J. P. Puel, Résultats d'existence pour certains problèmes elliptiques quasi-linéaires, Ann. Scuola Norm. Sup. Pisa Cl. Sci. 11 (1984), 213-235.

6. L. Boccardo, F. Murat and J. P. Puel, Existence de solutions failes pour deséqu-ations elliptiques quasi-linéaires à croissance quadratique, in: Nonlinear Partial Differential Equations and Their Applications, J. L. Lions and H. Brezis eds., Collège de France Seminar, Vol. IV, Research Notes in Math. 84, Pitman, London, 1983, pp. 19-73. 
Tsang-Hai Kuo

Department of Applied Mathematics, National Chiao Tung University, Hsinchu, Taiwan

E-mail: thkuo@math.nctu.edu.tw

Yeong-Ju Chen

Hsinchu High School, Hsinchu, Taiwan

E-mail: u8622501@cc.nctu.edu.tw 